\title{
Can Fluxionality of Subnanometer Cluster Catalysts Solely Cause Non- Arrhenius Behavior in Catalysis?
}

\author{
Borna Zandkarimi ${ }^{a}$ and Anastassia N. Alexandrova ${ }^{a, b^{*}}$ \\ aDepartment of Chemistry and Biochemistry, University of California, Los Angeles, Los \\ Angeles, CA 90095

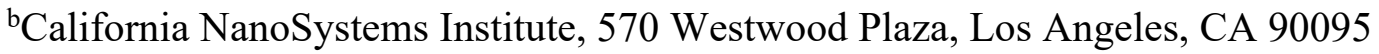

SUPPORTING INFORMATION

*Corresponding Author: Anastassia N. Alexandrova, (310) 825-3769, ana@chem.ucla.edu 

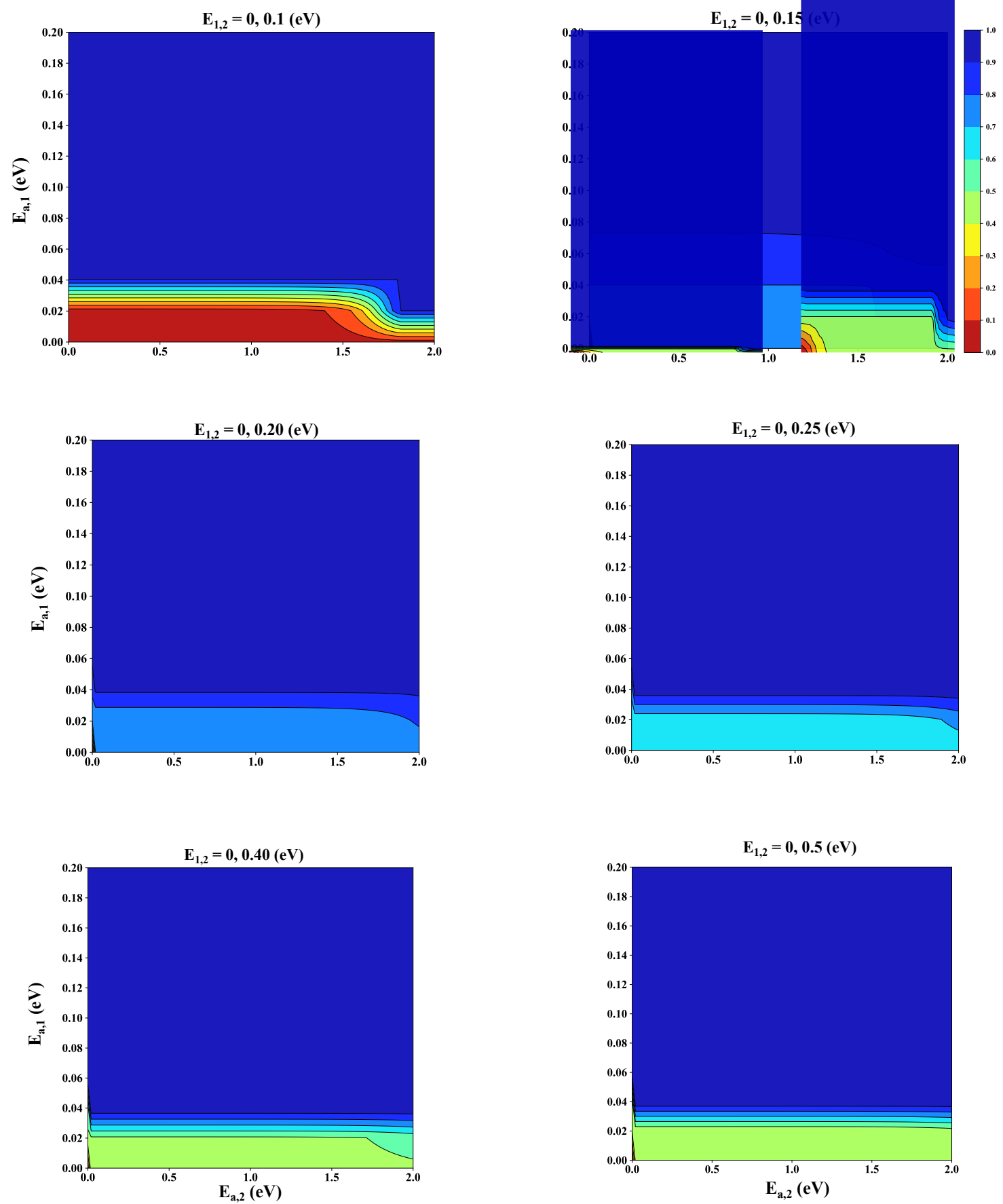

Figure S1. $R^{2}$ value obtained from 40,000 different combinations of $\mathrm{E}_{\mathrm{a}, 1}$ and $\mathrm{E}_{\mathrm{a}, 2}$ in the range of $0-2 \mathrm{eV}$ for the ensemble of two isomers with different relative energies $E_{1}$ and $E_{2}$ shown above each plot. The temperature range in which $R^{2}$ is calculated is $300-1000 \mathrm{~K}$.. Note that only the interesting region of $\mathrm{E}_{\mathrm{a}, 1}$ $=0-0.2 \mathrm{eV}$ (with low $R^{2}$ ) is shown here. 
Table S1. Change in the slope of $\ln k_{\text {ens }}$ vs. $1 / T$ plot corresponding to Figure 2 over the first and last $50 \mathrm{~K}$ temperature range.

\begin{tabular}{ccccccc}
\hline \multicolumn{7}{c}{ Slope $\frac{1}{k_{B}}\left(\left\langle E_{T S}\right\rangle-\left\langle E_{R}\right\rangle\right)$} \\
\hline $\mathrm{T}(\mathrm{K})$ & Ens 1 & Ens 2 & Ens 3 & Ens 4 & Ens 5 & Ens 6 \\
\hline $300-350$ & -44.8001 & -56.4046 & -102.8232 & -160.8600 & -276.8898 & -625.1700 \\
$950-1000$ & 276.2990 & 264.9550 & 218.3788 & 160.1700 & 44.2700 & -303.9266 \\
\hline$\Delta$ Slope (K) & 321.1 & 321.4 & 321.2 & 321.03 & 321.16 & 321.24 \\
$\Delta$ Slope (eV) & 0.0277 & 0.0277 & 0.0277 & 0.0277 & 0.0277 & 0.0277
\end{tabular}

